\title{
Evaluation du coefficient de diffusion du phosphore en sol de limon argileux calcaire
}

\author{
Robert HABIB \& Roger GUENNELON \\ I.N.R.A., Station de Science du Sol, Centre de Recherches agronomiques d'Avignon, B.P. 91, F 84140
} Montfavet.

\section{RÉSUMÉ}

Fertilisation,

Irrigation,

Solutés,

Transferts.
L'utilisation des engrais dans l'cau d'irrigation, plus spécialement dans le cas du goutte-à-goutte, cst conditionnéc par les propriétés respectives du sol et des solutés.

En sol argilo-calcaire, l'ion $\mathrm{PO}_{4}^{---}$est très énergiquement et rapidement insolubilisé et ses longueurs de trajet de diffusion sont très faibles. En utilisant ${ }^{32} \mathrm{P}$, on a mesuré le coefficient de diffusion pour diverses humidités et diverses densitćs apparentes. Sa valeur varic de 0,3 à $3,6 \cdot 10^{-7} \mathrm{~cm}^{2} \mathrm{~s}^{-1}$.

On a montré que, dans une gamme de teneurs en cau allant de 0,16 à 0,36 , on pouvait exprimer la tortuosité en fonction de l'indice des vides. Cette tortuosité étant appréciée ainsi, on peut calculer directement un coefficient de diffusion apparent, compte tenu des caractéristiques de la réaction d'insolubilisation et des niveaux de concentration de phosphates utilisés.
SUMMARY

Fertilization, Irrigation,

Solutes,

Transfers.

\section{Evaluation of the phosphorus diffusion coefficient of a calcareous clay loam soil}

Application of fertilizers in trickle irrigation water is closely dependent on the physical and chemical propertics of soil and solutes.

In a calcareous clay soil, the $\mathrm{PO}_{4}^{---}$ion does not move over long distances before becoming insoluble as $\mathrm{Ca}$ phosphates. A formula has been proposed to calculate an apparent diffusion coefficient, based on the physical properties of the soil (water content, porosity and tortuosity) and also taking phosphate concentration into account. This coefficient varied between $0.3 \times 10^{-7}$ and $3.6 \times 10^{-7} \mathrm{~cm}^{2} \mathrm{~s}^{-1}$, over the range of moisture content from 0.2 to 0.45 and of bulk densities from 1.13 to 1.58 .

This calculated cocfficient has been compared with measurements made using ${ }^{32} \mathbf{P}$ as a tracer of $\mathbf{P}$ displacement.

\section{INTRODUCTION}

Dans la pratique de la fertilisation, l'ion phosphate pose souvent quelques difficultés; l'une d'elles résulte de l'augmentation des risques de colmatage des canalisations et des goutteurs lorsqu'on pratique l'irrigation localisée fertilisante ; un autre problème découle de la faible mobilité de $\mathrm{PO}_{4}^{3-}$, dans des sols où les conditions physico-chimiques conduisent rapidement à l'insolubilisation de cet anion. On peut donc supposer, en particulier en «ferti-irrigation», qu'il sera difficile d'amener les engrais phosphatés au niveau des horizons de sol activement exploités par les racines. C'est pourquoi RaUSCHKOLB et al. (1976), O'NeILl et al. (1979) proposent, entre autres choses, l'utilisation de composés organiques (sels de $\mathrm{Ca}$ et de $\mathrm{Na}$ de l'acide glycérophosphorique), non hydrolysés par l'eau comme l'orthophosphate et susceptibles de migrer davantage. De nombreux travaux ont été réalisés, qui concernent la migration de l'ion orthophosphate mais, en sols calcaires, peu de recherches prennent en compte tous les facteurs spécifiques à ce type de matériel pédologique et au mode d'apport de l'engrais (FATHI AMER et al., 1980).
Par ailleurs, les expériences de transfert portent généralement sur des colonnes de sol à remplissage isotrope et relativement continu: la structure initiale du sol en place n'est alors pas conservée (de CAMARGO et al., 1979) et, si la modélisation des phénomènes est plus aiséc, elle ne peut cependant pas mettre en évidence les rôles de l'hétérogénéité du système et de l'existence de cheminements préférentiels ; or, ces caractéristiques de circulation peuvent, lors des apports d'eau (précipitations ou irrigations), réaliser un transfert convectif qui concurrence alors suffisamment les cinétiques d'insolubilisation, pour amener vers les zones d'absorption racinaire, des ions $\mathrm{PO}_{4}^{3-}$ encore utilisables par la plante. En particulier, les travaux de PAssioura (1971) et, plus récemment, de de CAMARGO et al. (1979), montrent que l'augmentation de la vitesse moyenne dans les pores permet cette augmentation de la distance de transfert de l'orthophosphate. Les travaux théoriques de Van GENUCHTEN \& WIERENGA (1976) montrent, par simulation, l'influence de ce facteur et précisent les paramètres à déterminer pour utiliser leur modèle.

La connaissance des mécanismes d'insolubilisation de $\mathrm{PO}_{4}^{3-}$ et leur description quantitative sont des étapes 
fondamentales de la modélisation, ainsi que la détermination préalable du coefficient de diffusion dans le milieu considéré. Ces paramètres sont nécessaires à l'établissement de la forme de la fonction puits, à introduire dans l'ćquation de transfert, pour quantifier la diminution de la concentration en phosphates de la solution de transfert. En effet, lors d'un transfert convectif, dans des pores de grandes dimensions, les parois des pores sont le siège de mouvements latéraux diffusifs. Pour un anion tel que $\mathrm{NO}^{-}$, la quantité échappant ainsi au transfert peut, dans certaines conditions, atteindre 50 p. 100 de la quantité injectée (de COCKBORNE, 1980). Ce phénomène est sous la dépendance du coefficient de diffusion moléculaire dont on mesure ainsi l'importance, même au cours d'un flux convectif rapide.

Par ailleurs, peu d'expériences au champ mettent en évidence la possibilité de migration des orthophosphates consécutive à l'accélération des flux hydriques dus à l'absorption racinaire (GUENNELON et al., 1979).

Si l'on s'intéresse maintenant aux phénomènes physicochimiques responsables de l'insolubilisation des phosphates dans le sol, on note que la plupart des travaux portent sur des sols acides ou neutres, où l'on considère surtout l'adsorption de $\mathrm{P}$ sur les colloïdes du sol, et la précipitation par les cations $\mathrm{Al}^{3+}$ et $\mathrm{Fe}^{3+}$. En sols calcaires, l'abondance du cation $\mathrm{Ca}^{++}$, tant en solution que sous forme échangeable ou à l'état de carbonates, accroît les risques d'immobilisation de l'orthophosphate et rendrait son utilisation précaire, si les mécanismes d'accélération de flux hydrique cités précédemment ne pouvaient entrer en compétition avec les cinétiques de précipitation. Les processus d'insolubilisation de l'ion $\mathrm{PO}_{4}^{3-}$ des divers orthophosphates solubles par le $\mathrm{CaCO}_{3}$ ont été étudiés par de nombreux auteurs dont BoIsCHOT et al. (1950), ColE et al. (1953) (pour ne citer que les plus anciens) et une revue de ces recherches figure dans un travail de ARVIEU (1972), qui traite de l'étude de ces réactions.

Le but du présent article est de proposer l'utilisation de méthodes permettant de déterminer certains paramètres, nécessaires ultérieurement à la modélisation des transferts de $\mathrm{P}$, y compris dans le cadre de la «ferti-irrigation» phosphatée. Dans les modèles déjà élaborés par certains auteurs, ces paramètres, propres au soluté, sont les caractéristiques des isothermes d'adsorption (fonction puits) et le coefficient de diffusion moléculaire apparent, lequel dépend d'ailleurs, dans le cas de $\mathrm{PO}_{4}^{3-}$, de la réaction d'adsorption.

La méthodologie utilisée pour déterminer ces paramètres doit également permettre d'évaluer le sens de leur action sur le transfert.

\section{ASPECTS THÉORIQUES}

Dans un milieu riche en ions $\mathrm{Ca}^{++}$, les sels solubles de l'acide orthophosphorique vont subir des évolutions qui aboutiront finalement à des composés apatitiques insolubles (ARvIEU, 1972). Les mécanismes que l'on peut invoquer sont complexes; dans un premier temps, le $\mathrm{Ca}^{++}$de la solution du sol peut conduire à la formation de phosphate monocalcique ou bicalcique ; il en résulte un nouvel état d'équilibre avec le complexe adsorbant, par interaction de l'ion potassium (provenant du phosphate utilisé). Dans le sol, l'évolution ultérieure du phosphate monocalcique soluble peut être considérablement freinée, par la présence d'argile (BOUVIER, 1974). Par ailleurs, le phosphate monopotassique peut agir rapidement sur $\mathrm{CaCO}_{3}$ pour donner le phosphate bicalcique dihydraté avec dégagement de $\mathrm{CO}_{2}$, l'évolution ultérieure, apatitique, étant une réaction lente.
Ces schémas dépendent, en outre, des concentrations mises en jeu, et du $\mathrm{pH}$ initial du mélange. Il est clair que, lors d'études sur la diffusion, le fait de créer, en tant que condition aux limites, une source d'ions $\mathrm{PO}_{4}^{3-}$ relativement concentrée, induira toute une gamme de conditions de milieu qui donneront lieu à des types de réaction différents (au moins en ce qui concerne leur vitesse). Enfin, on ne peut négliger, parmi les phénomènes possibles, la cristallisation épigénique de phosphates de calcium sur les cristaux de calcite présents dans le sol.

On ne peut assimiler la réaction entre $\mathrm{PO}_{4}^{3-}$ et un sol calcaire à une simple précipitation où la quantité fixée nc dépendrait que de la concentration initiale en $\mathrm{PO}_{4}^{3-}$ et des produits de solubilité des produits insolubles obtenus. Les réactions de sels acides sur $\mathrm{CaCO}_{3}$ peuvent également être limitées par la pression partielle du $\mathrm{CO}_{2}$ produit par la réaction, si ce $\mathrm{CO}_{2}$ ne peut être rapidement évacué de l'endroit où elle se produit.

La réaction d'immobilisation de l'orthophosphate ne doit pas non plus être considérée comme l'équivalent d'une réaction d'équilibre d'échange ; elle ne permet que d'expliciter une relation entre la quantité d'ions en solution et celle qui a disparu de la solution pour s'intégrer d'une manière ou d'une autre à la phase solide. $\mathrm{Si} \mathrm{S}_{\mathrm{E}}$ est la quantité de phosphore ainsi insolubilisée (en $\mu \mathrm{g}$ par g de solide) et $\mathrm{C}_{\mathrm{E}}$ la concentration dans la solution en $\mu \mathrm{g}$ de $\mathrm{P}$ par $\mathrm{ml}$ de solution à l'équilibre, on obtiendra une relation : $S_{E}=f\left(C_{E}\right)$ que l'on introduira dans l'équation de diffusion du soluté :

$$
\frac{\partial \mathrm{C}_{\mathrm{E}}}{\partial \mathrm{t}}+\alpha \frac{\partial \mathrm{S}_{\mathrm{E}}}{\partial \mathrm{t}}=\mathrm{D} \frac{\partial^{2} \mathrm{C}_{\mathrm{E}}}{\partial \mathrm{x}^{2}}
$$

en respectant, par un paramètre $\alpha$ approprié, l'homogénéité dimensionnelle de l'équation; $\mathrm{D}$ est un coefficient de diffusion apparent tenant compte des propriétés géométriques du milieu.

Des mesures de $C_{E}$ en fonction de $t(s)$ et de $x(\mathrm{~cm})$, permettent de déterminer le coefficient de diffusion $\mathrm{D}$, pouvant être utilisé par la suite dans les modèles. HiRA \& SingH (1978) ont proposé une méthode permettant cette mesure à différentes teneurs en eau et pour des milieux à caractéristiques physiques différentes. Cette méthode s'appuie sur une solution de l'équation (1) donnée par CARSLAW \& JAEGER (1947); si l'on a pour l'isotherme d'absorption (d'après HIRA \& SiNG 1977) :

$$
\mathrm{S}=\mathrm{bC} \text {, }
$$

la solution de l'équation (1) est, avec :

$$
\mathrm{t}=0, \quad \mathrm{C}=0 \text { pour tout } \mathrm{x} \geqslant 0
$$

$$
C(t, x)=\frac{Q_{A}(b+1)^{1 / 2}}{(\pi D t)^{1 / 2}} \cdot \exp \left\{-\frac{x^{2}(b+1)}{4 D t}\right\}
$$

où $\mathrm{Q}_{\mathrm{A}}\left(\mathrm{mg}\right.$ de $\mathrm{P}$ par $\mathrm{cm}^{2}$ ) est la quantité de phosphore (sous forme orthophosphate), initialement déposé à la surface d'un milieu dans lequel il peut librement diffuser dans la direction $\mathrm{x}$, dans le cas d'un milieu semi-infini.

HIRA \& SING (1978), déterminant ainsi D, comparent les valeurs trouvées avec celles qu'ils calculent à partir d'une formule simple proposée par NYE (1968), et qui met en œuvre le coefficient de diffusion moléculaire du phosphore en solution $D_{0}$, le facteur de tortuosité $f$ (ou son inverse $\tau$ ), l'humidité volumique $\theta$ et la pente $b$ de l'isotherme d'adsorption :

$$
\mathrm{D}=\frac{\mathrm{D}_{0} \theta}{\mathrm{b} \tau}
$$


Notre but est de rechercher la cohérence des résultats, obtenus pour $\mathrm{D}$, déduits de nos expériences sur sol calcaire, avec les résultats calculés par la formule simplifiée de NYE et de mettre en évidence les propriétés physiques du milicu qui, en agissant sur D, modifient la mobilité de l'ion phosphate.

\section{MÉTHODES DE MESURE}

Le matériau sur lequel ont porté les expériences est l'horizon de surface $(0-30 \mathrm{~cm})$ d'un sol d'alluvions argilocalcaires dont les caractéristiques sont reportées au tableau 1.

\section{TABLEAU 1}

Caractéristiques physico-chimiques du sol utilisé. Physico-chemical composition of the soil.

\begin{tabular}{lr}
\hline Argile & 37.9 \\
Limon $2-20 \mu$ & 41.2 \\
$20-50 \mu$ & 10.9 \\
Sables $50-200 \mu$ & 7.7 \\
$\quad 200-2000 \mu$ & 2.2 \\
Matières organiques & 2.0 \\
Calcaire total & 34.1 \\
\hline
\end{tabular}

\section{A. Isotherme d'insolubilisation de $\mathrm{KH}_{2} \mathrm{PO}_{4}$}

On a agité pendant 3 jours, à $20^{\circ} \mathrm{C}, 32 \mathrm{~g}$ de sol et $32 \mathrm{ml}$ de solution de phosphate monopotassique marqué au ${ }^{32} \mathrm{P}$, pour diverses concentrations. Après centrifugation, on a mesuré $\mathrm{P}$ par comptage radiométrique dans le liquide surnageant. Dans une expérience préliminaire, on a vérifié que l'activité de la solution ne décroissait plus notablement après $48 \mathrm{~h}$ de contact et d'agitation avec le sol.

Les quantités «fixées » par le sol ont été déduites, par différence, des quantités présentes initialement dans la solution.

\section{B. Tortuosité}

On a déterminé la tortuosité avec de l'eau tritiée ce qui rend compte, en principe, des caractéristiques géométriques de l'espace poral correspondant le mieux à ce concept. Le tritium n'est en effet pas soumis à divers phénomènes d'exclusion ou d'adsorption qui modifieraient sa mobilité, la teneur en eau du milieu étant uniforme, sauf pour les sols riches en matières organiques; dans ce cas, il peut y avoir des échanges isotopiques de ${ }^{3} \mathrm{H}^{+}$avec des atomes d'hydrogène autres que ceux de l'eau.

On peut définir la tortuosité par l'expression :

$$
\tau_{3 \mathrm{H}}=\frac{\mathrm{D}_{0, \mathrm{H}}}{\mathrm{D}_{\mathrm{H}}}
$$

où $\mathrm{D}_{0, \mathrm{H}}$ est le coefficient (NAKAYAMA \& JACKSON, 1963) d'autodiffusion dans l'eau $\left(24,5 \cdot 10^{-6} \mathrm{~cm}^{2} \mathrm{~s}^{-1}\right)$ et $\mathrm{D}_{\mathrm{H}}$ le coefficient de l'eau tritiée dans le milieu considéré. La méthode consiste à mesurer $D_{H}\left(D_{0, \mathrm{H}}\right.$ étant connu pour une valeur moyenne de la concentration en soluté ou en traceur). On peut appliquer l'équation générale de diffusion à une situation déterminée, qui impose alors les conditions aux limites et les conditions initiales nécessaires à la résolution de l'équation.
Le mode opératoire est celui utilisé par HIRA \& SINGH (1977) ; deux échantillons de sol identiques, l'un en équilibre avec de l'eau tritiée, l'autre avec de l'eau non marquée, sont placés dans 2 compartiments identiques de longueur $L$. Le tritium diffuse alors dans le second compartiment.

Un tel dispositif réalise des conditions initiales et aux limites de résolution de l'équation de diffusion, dont l'expression a été donnée par SCHOFIELD \& GRAHAMBRYCE (1960) :

$$
\begin{aligned}
\frac{\overline{\mathrm{C}}}{\mathrm{C}_{\infty}}=1-\frac{8}{\bar{\Pi}^{2}} \sum_{\mathbf{k}=0}^{\infty} & \frac{1}{(2 \mathrm{k}+1)^{2}} \times \\
& \times \exp \left\{-\frac{(2 \mathrm{k}+1)^{2} \Pi^{2}}{\mathrm{~L}^{2}} \mathrm{D}_{\mathrm{H}} \mathrm{t}\right\}
\end{aligned}
$$

où $\overline{\mathrm{C}}$ est la concentration moyenne en élément diffusant au bout du temps $\mathrm{t}$, dans le second compartiment de diffusion.

$\mathrm{C}_{x}$ est la concentration moyenne finale, soit la moitié de la concentration initiale $\mathrm{C}_{0}$. Ces concentrations sont exprimées par l'activité de ${ }^{3} \mathrm{H}$.

La diffusion est réalisée pendant 1 à $4 \mathrm{j}$ selon l'activité en tritium introduite, à température constante de $20^{\circ} \mathrm{C}$. Si $\mathrm{N}_{1}$ et $\mathrm{N}_{2}$ sont des grandeurs proportionnelles à la quantité de ${ }^{3} \mathrm{H}$ contenue dans chacune des 2 cellules, on a :

$$
\frac{\overline{\mathrm{C}}}{\mathrm{C}_{\infty}}=2 \frac{\mathrm{N}_{2}}{\mathrm{~N}_{1}+\mathrm{N}_{2}} \text {. }
$$

Pour chaque couple de valeurs des paramètres expérimentaux : $H$ (humidité massique) et $\rho_{\mathrm{d}}$ (masse volumique sèche), on a réalisé 8 répétitions et la formule de SCHOFIELD a été utilisée par sommation jusqu'à $\mathrm{k}=2$, la convergence à cette valeur étant estimée pour un résidu de $\pm 0,001$ (pour $\mathrm{C}_{x}$ variant de 0,7 à 0,9 ).

\section{Coefficient de diffusion de l'orthophosphate}

\section{1) Mode opératoire}

Des échantillons d'un sol, amenés à la même humidité, sont tassés dans 2 cellules cylindriques de $30 \mathrm{~mm}$ de diamètre et de $40 \mathrm{~mm}$ de longueur. On réalise dans chacune d'elles des densités identiques par déplacement d'un piston.

Une quantité connue de cristaux très finement broyés de $\mathrm{KH}_{2} \mathrm{PO}_{4}$ marqués au ${ }^{32} \mathrm{P}$ est répandue aussi uniformément que possible sur la surface de l'une de ces cellules. Ces cellules sont ensuite accolées, en contact par cette surface, et la jonction est paraffinée pour éviter des modifications de teneur en eau. Au bout d'un temps déterminé, à température contrôlée de $20^{\circ} \mathrm{C}$, les 2 cellules sont séparées et, la diffusion étant supposée symétrique, on échantillonne l'une d'elles, millimètre par millimètre. Ces prélèvements de sol sont séchés à l'air et finement broyés ; un poids connu de sol est étalé dans des coupelles de $80 \mathrm{~mm}$ de diamètre et compté pour ${ }^{32} \mathrm{P}$ dans un compteur à flux gazeux. On a réalisé avec le même sol une gamme de comparaison avec des solutions de concentrations connues, pour la même activité spécifique par rapport à $\mathrm{P}$ que le $\mathrm{KH}_{2} \mathrm{PO}_{4}$ utilisé lors des essais. Compte tenu du faible poids des échantillons (de l'ordre de $1 \mathrm{~g}$ pour une densité de solide de 2,713) et de la surface de la coupelle, les corrections d'auto-absorption n'ont pas été nécessaires, les variations d'épaisseur entre échantillons à mesurer et échantillons de la gamme étant pratiquement nulles. On mesure ainsi des activités au ${ }^{32} \mathrm{P}$ que l'on rapporte à des concentrations en phosphore, calculées en $\mathrm{mg}$ de $\mathrm{P}$ par $\mathrm{cm}^{3}$ de sol en place dans la cellule 
de diffusion en tenant compte de la densité de remplissage (fig. 1). Des détails complémentaires sur cette technique peuvent être trouvés dans l'article de HIRA \& SiNGH (1978).

\section{2) Résolution de l'équation de diffusion}

Quelques ambiguités existant dans cet article au niveau des équations aux dimensions de la solution proposéc, nous établirons celle que nous avons utilisée pour nos calculs, en nous référant à la solution théorique donnée par CRANK (1956).

Soit :

$$
\mathrm{S}_{0}=\mathrm{bC}_{0}
$$

une expression qui relic, à l'équilibre, la quantité $\mathrm{S}_{0}$, associée à la phase solide, à la concentration $\mathrm{C}_{0}$ dans la solution. Déterminée par des expériences de mise en contact d'un poids donné de sol avec un volume donné de solution (cf. III, A), $\mathrm{S}_{0}$ est une masse de $\mathrm{P}$ par $\mathrm{g}$ de solide $\left(\mathrm{g} \mathrm{g}^{-1}\right)$ et $\mathrm{C}_{0}$ une masse par volume de solution $\left.(\mathrm{g} \mathrm{m}]^{-1}\right)$. Le coefficient «b » a pour dimension: $\mathrm{M}^{-1} \mathrm{~L}^{3}$.

L'équation de diffusion s'écrit :

$$
\frac{\partial \mathrm{C}_{0}}{\partial \mathrm{t}}+\frac{\rho_{\mathrm{d}}}{\theta} \frac{\partial \mathrm{S}_{0}}{\partial \mathrm{t}}=\mathrm{D}_{\mathrm{P}} \frac{\partial^{2} \mathrm{C}_{0}}{\partial \mathrm{x}^{2}}
$$

$\rho_{\mathrm{d}}$ étant la masse volumique du sol en $\left.\mathrm{g} \cdot \mathrm{m}\right]^{-1}, \theta$ l'humidité volumique $\left(\mathrm{cm}^{3} \mathrm{~cm}^{-3}\right)$ et $D_{\mathrm{P}}$ le coefficient de diffusion moléculaire de $\mathrm{PO}_{4}^{3-}$ dans le milieu considéré, indépendamment des phénomènes d'insolubilisation.

La concentration volumique, dans la cellule de diffusion, est donnée par :

$$
\mathrm{C}=\mathrm{C}_{0} \theta+\mathrm{S}_{0} \rho_{\mathrm{d}}=\mathrm{C}_{0}\left(\boldsymbol{\theta}+\mathrm{b} \cdot \rho_{\mathrm{d}}\right)
$$
(9):

L'équation (8) devenant après combinaison avec (7) et

$$
\frac{\partial \mathrm{C}}{\partial \mathrm{t}}=\left(\mathrm{D}_{\mathrm{P}} /\left(1+\frac{\rho_{\mathrm{d}} \mathrm{b}}{\theta}\right)\right) \frac{\partial^{2} \mathrm{C}}{\partial \mathrm{x}^{2}}
$$

Si $Q_{A}$ est la quantité totale de phosphore apporté à la jonction des 2 cellules, par unité de surface, la solution de cette équation est (CRANK, 1956):

$$
C=\frac{Q_{A}}{2} \cdot\left(\frac{\theta+b \rho_{d}}{\Pi \theta D_{p} t}\right)^{1 / 2} \cdot \exp \left\{-\frac{x^{2}\left(\theta+b \rho_{d}\right)}{4 \theta D_{p} t}\right\} \text {. }
$$

Posons :

$$
\mathrm{D}_{\mathbf{a}}^{*}=\mathrm{D}_{\mathrm{P}} /\left(1+\frac{\rho_{\mathrm{d}} \mathrm{b}}{\theta}\right)
$$

$\mathrm{D}_{\mathrm{a}}^{*}$ est le coefficient de diffusion apparent mesuré.

L'équation (11) s'écrit alors :

$$
C=\frac{Q_{\mathrm{A}}}{2} \cdot \frac{1}{\left(\Pi D_{\mathrm{a}}^{*} \mathrm{t}\right)^{1 / 2}} \cdot \exp \left\{-\frac{\mathrm{x}^{2}}{4 \mathrm{D}_{\mathrm{a}}^{*} \mathrm{t}}\right\}
$$

Un graphe de $\log C$ en fonction de $x^{2}$, pour une durée d'expérience donnée, sera représenté par une droite (fig. 2) de pente $m$.

On aura ainsi :

$$
\mathrm{D}_{\mathrm{a}}^{*}=\frac{-1}{4 \mathrm{mt}}
$$

L'erreur sur $D_{a}^{*}$ ne dépend donc que de l'erreur faite sur la pente lors de l'ajustement.

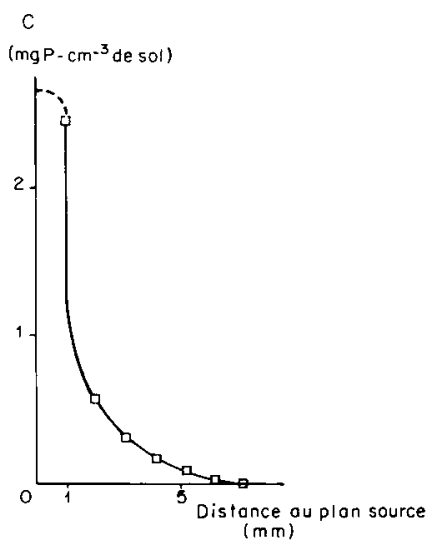

Figure 1

Exemple de distribution des concentrations en $P$ du sol en fonction de la distance au plan source.

Distribution of $P$ concentrations in the soil according to distance from the source.

Conditions expérimentales

(experimental conditions)

$\theta=0,42 ; \rho_{d}=1,57 ; Q_{A}=0,99$ mg.cm $\mathrm{cm}^{-2}$

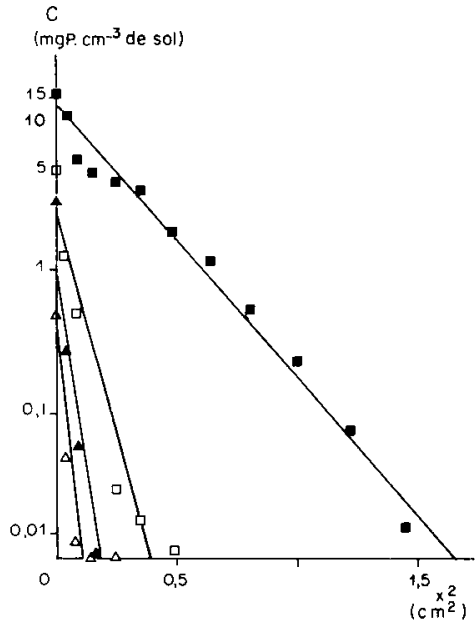

Figure 2

Représentation semi-logarithmique de la fonction $C\left(x^{2}, t\right)$ pour diverses valeurs de $Q_{A}$.

Semi-log representation of $C\left(x^{2}, t\right)$ for some values of $Q_{A}(P$ concentrations).

$\begin{array}{ll}\text { : }: 10 \mathrm{mg} \cdot \mathrm{cm}^{-2} & \square: 1,42 \mathrm{mg} \cdot \mathrm{cm}^{-2} \\ \Delta: 0,35 \mathrm{mg} \cdot \mathrm{cm}^{-2} & \Delta: 0,14 \mathrm{mg} \cdot \mathrm{cm}^{-2}\end{array}$

\section{RÉSULTATS ET INTERPRÉTATIONS}

\section{A. Réaction d'insolubilisation}

\section{1) Résultats}

Nous avons vu que toute équation rendant compte de la diffusion du phosphate devait comporter un terme exprimant la diminution de concentration de la solution par une réaction sol-soluté. Cette réaction est, dans le cas de $\mathrm{PO}_{4}^{3-}$, complexe puisque SELIM et al. (1976), en sols non calcaires, proposent un modèle à 2 sites d'échange, l'un immédiat, l'autre lent. On a vu qu'en sols calcaires, il faut tenir compte de la présence des ions $\mathrm{Ca}^{++}$en solution, adsorbés sur l'argile ou des réactions avec $\mathrm{CaCO}_{3}$. C'est pourquoi l'on a 
déterminé le coefficient de partage entre le sol et la solution avec un rapport sol/solution de $1 \mathrm{~g} . \mathrm{ml}^{-1}$, pour obtenir une relation $S_{0}=f\left(C_{0}\right)$ valable pour une humidité volumique compatible avec celles rencontrécs au champ lors de l'irrigation localisée (fig. 3). Cette fonction présente dcux parties que nous n'avons pas cherché à interpréter mais que, d'un point de vue opérationnel, nous avons assimilées à une droite obtenue par ajustement linéaire, d'équation :

$$
\mathrm{S}_{0}=9,92 \mathrm{C}_{0}
$$

Ic coefficient angulaire pouvant, dans la pratique, être pris égal à 10 , pour'ce type de sol. Les concentrations à l'équilibre, pour lesquelles ce coefficient s'écarte peu de la pente expérimentale, sont celles qui sont supérieures à $20 \mathrm{mg} / \mathrm{l}$, soit $300 \mathrm{mg} / \mathrm{l}$ environ pour la concentration initiale. Or, pour une fertilisation de $30 \mathrm{~kg}$ de $\mathrm{P}$ à l'ha, correspondant en moyenne à $50 \mathrm{~g}$ par arbre, apportés dans l'eau d'irrigation, on réalise, pour une E.T.P. (Evapo-transpiration potentielle) moyenne de $7 \mathrm{~mm} / \mathrm{j}$ des apports d'eau journaliers moyens, avec un débit de l'ordre de $61 / \mathrm{h}$ pendant $12 \mathrm{~h}$.

Si la fertilisation est apportée en 4 fois (soit $12,5 \mathrm{~g}$ en un jour), on aboutit à des concentrations initiales de :

- $173 \mathrm{mg} / \mathrm{l}$ si la durée d'injection est de $12 \mathrm{~h}$,

- $\quad 346 \mathrm{mg} / \mathrm{l}$ si cette durée est de $6 \mathrm{~h}$,

- $2076 \mathrm{mg} / \mathrm{l}$ si elle est de $1 \mathrm{~h}$.

Ces concentrations initiales correspondent à des concentrations à l'équilibre environ 10 fois plus faibles (de 17 à $207 \mathrm{mg} / \mathrm{l}$ ) : zone matérialisée sur la figure 3 . On voit donc que c'est dans cette zone que les écarts entre les valeurs réelles et celles de l'ajustement seront les plus variables. Lors de l'utilisation de cette fonction $S_{0}$ (cf. IV, C), on discutera de l'éventuelle prise en compte de ces écarts.

\section{2) Discussion}

Les résultats ainsi obtenus aboutissent donc à un isotherme traduisant une réaction équilibrée, à première vue incompatible avec une réaction totale de précipitation du phosphate sous forme apatitique. Cet état de fait peut provenir non seulement des mécanismes plus complexes que la simple précipitation, mais aussi des conditions expérimentales : en effet, le contact, avec agitation, du sol et de la

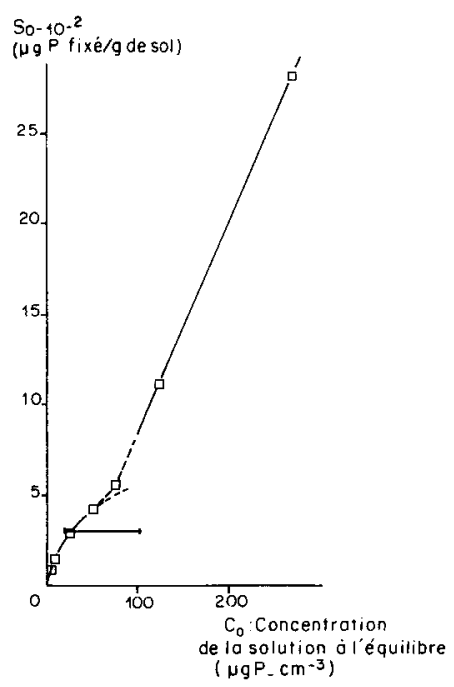

Figure 3

Isotherme traduisant la réaction "sol-soluté ".

Distribution of $P$ between solid and liquid phases. solution a lieu en flacons bouchés ; s'il y a production de $\mathrm{CO}_{2}$, celui-ci ne peut se dégager, ce qui limite la précipitation du phosphate bicalcique.

Dans le dispositif expérimental permettant de déterminer $D_{a}^{*}$, lc système «sol-solution du sol-orthophosphate » est également un système fermé, pour éviter des modifications de la teneur en eau. On a donc bien des conditions similaires, si l'influence de $\mathrm{CO}_{2}$ doit être mise en cause.

Pour un sol irrigué (ou un dispositif simulant un déplacement), il n'en scra peut-être plus de même et une toute autre méthodologie devrait être proposée (cf. IV, C, 1).

\section{B. Variations de la tortuosité en fonction des caractéristi- ques du milieu}

Les mesures de la tortuosité $\tau$ par la méthode précédemment exposée ont été faites pour diverses humidités massiques $\mathbf{H}\left(\mathrm{g} \cdot \mathrm{g}^{-1}\right)$ et densités apparentes $\rho_{\mathrm{d}}$ (tabl. 2).

Cette méthode étant d'une mise en œuvre peu aisée, on peut chercher, pour un sol donné, à relier statistiquement $\tau$, aux caractéristiques physiques qui agissent sur le coefficient de diffusion. Pour des teneurs en eau telles que les pores interagrégats les plus fins soient toujours saturés (assurant ainsi une certaine continuité des veines liquides), on peut admettre que la tortuosité est d'autant plus faible que l'indice des vides $\frac{\rho_{\mathrm{s}}-\rho_{\mathrm{d}}}{\rho_{\mathrm{d}}}\left(\rho_{\mathrm{s}}\right.$ : densité de solide) est plus élevé, c'est-à-dire que le volume occupé par le solide est faible par rapport au volume des pores.

On peut donc proposer une relation où $\tau$ est fonction de $\frac{\rho_{\mathrm{d}}}{\rho_{\mathrm{s}}-\rho_{\mathrm{d}}}$. En considérant les valeurs moyennes figurant au tableau 2, on arrive à un ajustement :

$$
\tau=a\left(\frac{\rho_{d}}{\rho_{s}-\rho_{d}}\right)^{B}
$$

avec $\mathrm{a}=2,69$ et $\mathrm{B}=0,97$, pour un coefficient de corrélation $\mathrm{r}=0,96\left(^{* *}\right)$ (fig. 4 ).

On peut voir que $\tau$ atteint la valeur 1 pour une valeur $\rho_{d}$ minimale qui devrait correspondre à un assemblage peu cohérent.

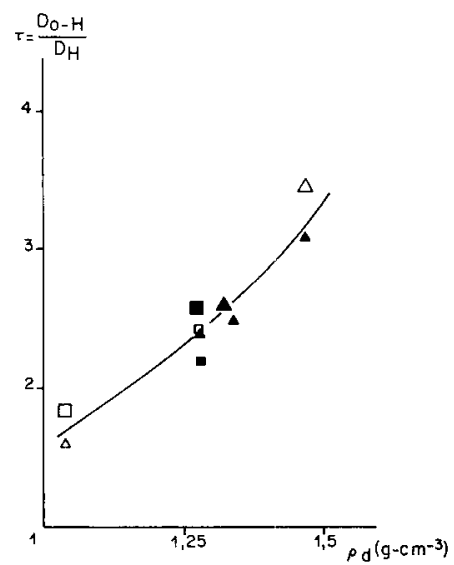

Figure 4

Variation de la tortuosité en fonction de la masse volumique du sol pour différentes valeurs de teneur en eau $H$.

Tortuosity data for various moisture contents versus bulk density. Water content \%.
A : $0,20 \mathrm{~g} \cdot \mathrm{g}^{-1}$
A: $0,29 \mathrm{~g} \cdot \mathrm{g}^{-1}$
ㅁ: $0,33 \mathrm{~g} \cdot \mathrm{g}^{-1}$
: $0,38 \mathrm{~g} \cdot \mathrm{g}^{-1}$
$\triangle: 0,21 \mathrm{~g} \cdot \mathrm{g}^{-1}$
$\triangle: 0,31 \mathrm{~g} \cdot \mathrm{g}^{-1}$
: $0,37 \mathrm{~g} \cdot \mathrm{g}^{-1}$ 
Les teneurs en eau choisies ne correspondent pas à des valeurs limitantes, pour lesquelles la tortuosité augmenterait notablement quand l'humidité diminuerait.

Ces résultats sont en accord avec ceux trouvés par HiRA \& SiNGH (1977) qui ne montrent des variations significatives pour 2 types de sol qu'aux faibles densités pour des teneurs en eau de 7 à 18 p. 100 , et des variations moindres pour des humidités allant de 14 à 25 p. 100 .

\section{Valeurs obtenues pour le coefficient de diffusion de $\mathrm{PO}_{4}^{3-}$}

Le tableau 2 donne les résultats obtenus pour 12 ensembles de valeur des paramètres: humidité volumique $\theta$, masse volumique du sol sec $\rho_{d}$ ct masse de phosphore apporté $Q_{A}$. Les valeurs de la tortuosité indiquées ont été calculées à partir des valeurs de $\rho_{\mathrm{d}}$ par la relation (16).

\section{1) Utilisation de la solution de l'équation de diffusion}

La transformation logarithmique de la formule (13) a permis de calculer, en utilisant la pente de la transformée, les valeurs $D_{a}^{*}$ du coefficient de diffusion apparent du $\mathrm{PO}_{4}^{---}$(tabl. 2).

$\mathrm{Ce}$ coefficient est inférieur d'un ordre de grandeur seulement à celui du coefficient d'autodiffusion dans l'cau $\left(D_{0, \mathrm{P}}=8,9 \cdot 10^{-6} \mathrm{~cm}^{2} \cdot \mathrm{s}^{-1}\right)$, mais de deux ordres de grandeur à celui du tritium $\left(D_{0, \mathrm{H}}=2,5 \cdot 10^{-5} \mathrm{~cm}^{2} \cdot \mathrm{s}^{-1}\right)$. Notons que $D_{a}^{*}$ a été déterminé expérimentalement sans faire intervenir les paramètres $b$ et $\tau$ déterminés précédemment. Néanmoins, 2 hypothèses ont été nécessaires pour cette détermination :

- d'une part, le coefficient de diffusion moléculaire $D_{p}$ a été supposé indépendant de la concentration en $\mathrm{PO}_{4}^{3-}$. Or cette concentration varie le long de la courbe de distribution de $\mathrm{P}$ dans le milieu. Peu de mesures sont disponibles sur cette variation de $D_{p}$. En fait, pour d'autres sels comme $\mathrm{KCl}, \mathrm{NaCl}$ ou $\mathrm{KNO}_{3}$ la variation relative est de l'ordre de 10 p. 100 quand on passe d'une concentration quasi nulle à une concentration $5 \cdot 10^{-2} \mathrm{M}$. Or les concentrations rencon- trées dans nos essais sont toutes inférieures à $10^{-4} \mathrm{M}$, sauf au voisinage du plan source ;

- d'autre part, on a supposé, pour établir l'équation (13), que la relation entre $S_{0}$ et $C_{0}$ était linéaire (b constant); on voit sur la figure 3 qu'il n'en est rien ; une amélioration de la méthode passe donc par la décomposition de la fonction $S_{0}$ en une relation du type $S_{0}=K C_{0}^{\mathrm{N}}$ suivie d'une relation linéaire. La résolution de l'équation (8) doit alors faire l'objet d'un traitement numérique ou d'une solution analytique plus complexe. Le calcul de $\mathrm{D}_{\mathbf{a}}^{*}$ devrait alors être effectué par ajustement non linéaire ;

- enfin, dans plusieurs cas, le nombre de points expérimentaux obtenus pour la courbe de diffusion, est trop faible pour que la précision sur $\mathrm{D}_{\mathrm{a}}^{*}$ soit bonne (tabl. 2).

2) Estimation du coefficient de diffusion à partir des caractéristiques du milieu

Considérant le flux de diffusion dans un milieu poreux comme proportionnel à l'humidité du sol, au facteur de tortuosité $\left(\mathrm{f}=\frac{1}{\tau}\right)$ et à l'état de l'équilibre $\frac{\mathrm{dS}}{\mathrm{dC}}$ entre la phase solide et la phase liquide, quant à l'ion étudié, NYE (1966) a proposé la formule :

$$
\mathrm{D}_{\mathrm{a}, 1}=\mathrm{D}_{0, \mathrm{P}} \frac{\theta}{\tau \mathrm{b}}
$$

où b est la valeur de $\frac{\mathrm{d} S}{\mathrm{dC}}$, l'isotherme d'adsorption étant supposé linéaire, $\mathrm{S}$ et $\mathrm{C}$ étant exprimés tous deux en masse par volume (de sol et de solution). Il s'agit alors d'un coefficient apparent propre au sol, indépendant de la quantité de soluté soumis à la diffusion (RowEll et al., 1967).

HIRA \& Singh (1978), pour tenir compte de la quantité d'élément susceptible de diffuser et d'agir sur les gradients de concentration, ont proposé :

$$
\mathrm{D}_{\mathrm{a}, 2}=\mathrm{D}_{0, \mathrm{P}} \frac{\theta}{\tau \mathrm{b}}(\mathrm{Q})^{1 / 2}
$$

TABLEAU 2

Mesure du coefficient de diffusion apparent de l'ion $\mathrm{PO}_{4}^{3-}$ pour différentes conditions.

\begin{tabular}{|c|c|c|c|c|c|c|c|c|c|c|c|c|}
\hline$N^{\circ}$ de l'essai & 1 & 2 & 3 & 4 & 5 & 6 & 7 & 8 & 9 & 10 & 11 & 12 \\
\hline$\theta\left(\mathrm{cm}^{3} \cdot \mathrm{cm}^{-3}\right)$ & 0,23 & 0,23 & 0,23 & 0,23 & 0,26 & 0,27 & 0,38 & 0,41 & 0,42 & 0,42 & 0,45 & 0,45 \\
\hline$\rho_{\mathrm{d}}\left(\mathrm{g} \cdot \mathrm{cm}^{-3}\right)$ & 1,13 & 1,13 & 1,13 & 1,13 & 1,28 & 1,34 & 1,41 & 1,49 & 1,57 & 1,58 & 1,50 & 1,50 \\
\hline Tortuosité & 1,90 & 1,90 & 1,90 & 1,90 & 2,40 & 2,60 & 2,90 & 3,20 & 3,70 & 3,70 & 3,30 & 3,30 \\
\hline $\mathrm{Q}_{\mathrm{A}}\left(\mathrm{mg} \cdot \mathrm{cm}^{-2}\right)$ & 0,14 & 0,35 & 1,42 & 10,0 & 10,0 & 10,0 & 0,99 & 0,99 & 0,99 & 12,9 & 12,9 & 10,3 \\
\hline Temps (h) & 49,5 & 52,1 & 49,7 & 99,0 & 99,0 & 117,4 & 90,7 & 91,3 & 93,5 & 123,0 & 120,0 & 69,4 \\
\hline Pente : - m & 44,7 & 36,9 & 14,3 & 4,39 & 5,85 & 5,60 & 28,2 & 15,5 & 15,6 & 1,59 & 1,59 & 3,57 \\
\hline$D^{*} 10^{7}\left(\mathrm{~cm}^{2} \cdot \mathrm{s}^{-1}\right)$ & 0,3 & 0,4 & 1,0 & 1,6 & 1,2 & 1,0 & 0,3 & 0,5 & 0,5 & 3,5 & 3,6 & 2,8 \\
\hline Intervalle & 0,6 & 0,8 & 1,3 & 1,7 & 1,3 & 1,3 & 0,4 & 0,6 & 0,7 & 3,9 & 4,0 & 3,1 \\
\hline $\begin{array}{c}\text { de confiance } \\
(\alpha=0,05)\end{array}$ & $\overline{0,2}$ & $\overline{0,2}$ & $\overline{0,7}$ & $\overline{1,3}$ & $\overline{1,0}$ & $\overline{0,8}$ & $\overline{0,2}$ & $\overline{0,3}$ & $\overline{0,3}$ & $\overline{3,0}$ & $\overline{3,1}$ & $\overline{2,3}$ \\
\hline
\end{tabular}
Measured values of apparent diffusion coefficient of $\mathrm{PO}_{4}^{3-}$ for different conditions. 
Le facteur $Q$ est le rapport $Q_{A} / Q_{i}, Q_{i}$ (quantité unitaire) étant la valeur de $Q_{A}$ pour laquelle leurs résultats expérimentaux se rapprochent le plus des valeurs calculées à partir de la formule de NYE.

Les résultats calculés par cette formule pour les 12 expériences précédentes figurent au tableau 3 .

Les modifications ainsi apportées à la formule (17) n'améliorent pas complètement la relation entre les coefficients ainsi calculés et les coefficients mesurés. L'influence de la quantité de sel apporté sur la section de l'échantillon ne dépend pas seulement de cette quantité ; même si le phosphate de potassium est réparti sur toute la surface, on peut admettre que ne participe directement à la diffusion que la quantité de sel qui se trouve face à la fraction de porosité occupée par de l'eau. Un cristal de sel situé contre un agrégat ou un grain de solide sera moins " efficace » que s'il se trouve engagé dans un pore rempli de liquide.

On a donc cherché à relier cette efficacité $\mathrm{E}$ à la porosité n et au taux de saturation $\theta_{s}=\theta / n$.

$$
\mathrm{D}_{\mathrm{a}}^{*}(\text { mesuré })=\mathrm{D}_{\mathrm{a}, 1} \cdot \mathrm{E}
$$

Ce coefficient d'efficacité de l'apport aura les caractéristiques suivantes:

- il doit exprimer que cette efficacité est potentiellement proportionnelle au volume des vides (c'est-à-dire, dans le plan source), à la surface apparente des vides par rapport à la surface totale : on introduit ainsi la porosité «n $n$.

- il doit être tel que, si le taux de saturation $\theta_{\mathrm{s}}$ est de 100 p. 100 , l'efficacité n'est proportionnelle qu'à la quantité apportée et à la porosité.

Ayant déterminé $\mathrm{E}$ pour les 12 expériences par la formule (19), on a cherché à ajuster ces valeurs (tabl. 4) à une relation $\mathrm{f}\left(\boldsymbol{\theta}_{\mathrm{s}}, \mathrm{n}\right.$ et $\left.\mathrm{Q}_{\mathrm{A}}\right)$. On propose ainsi une relation empirique :

$$
E=n\left(10^{3} \bar{Q} A\right)^{\theta_{s}}
$$

$\mathrm{n}$ et $\theta_{\mathrm{s}}$ étant sans dimension et $\overline{\mathrm{Q}}_{\mathrm{A}}$ étant la valeur numérique de la quantité apportée (en $\mathrm{g} \cdot \mathrm{cm}^{-2}$ ). Cherchant un coefficient d'ajustement, on n'affectera pas à $\bar{Q}_{\mathrm{A}}$ sa dimension propre.

\section{TABLEAU 3}

Estimation du coefficient de diffusion apparent selon les équations (17), (18) et (21).

Estimated values of apparent diffusion coefficient according to equations (17), (18) and (21).

\begin{tabular}{rcccc}
\hline \hline $\begin{array}{c}N^{\circ} \text { de } \\
\text { l'essai }\end{array}$ & $\begin{array}{c}\mathrm{D}_{\mathrm{a}}^{*} \times 10^{7} \\
\text { mesuré }\end{array}$ & $\begin{array}{c}\mathrm{D}_{\mathrm{a}, 1}: \\
\text { équation } 17\end{array}$ & $\begin{array}{c}\mathrm{D}_{\mathrm{a}, \mathrm{i}} \text { calculé } \times 10^{7} \\
\mathbf{D}_{\mathrm{a}, 2}: \\
\text { équation } 18\end{array}$ & $\begin{array}{c}\mathbf{D}_{\mathrm{a}, 3}: \\
\text { équation 21 }\end{array}$ \\
\hline 1 & 0,3 & 0,94 & 0,35 & 0,25 \\
2 & 0,4 & 0,94 & 0,57 & 0,36 \\
3 & 1,0 & 0,94 & 1,12 & 0,63 \\
4 & 1,6 & 0,94 & 2,59 & 1,35 \\
5 & 1,2 & 0,76 & 2,39 & 1,24 \\
6 & 1,0 & 0,69 & 2,18 & 1,18 \\
7 & 0,3 & 0,83 & 0,83 & 0,40 \\
8 & 0,5 & 0,76 & 0,75 & 0,34 \\
9 & 0,5 & 0,66 & 0,65 & 0,28 \\
10 & 3,5 & 0,64 & 2,30 & 3,42 \\
11 & 3,6 & 0,82 & 2,93 & 4,69 \\
12 & 2,8 & 0,82 & 2,62 & 3,64 \\
\hline \hline
\end{tabular}

TABLEAU 4

Valeurs mesurées et calculées du coefficient d'efficacité $E$. Measured and calculated values for the efficiency coefficient $E$.

\begin{tabular}{rcccc}
\hline \hline & $\begin{array}{c}\mathrm{D}_{\mathrm{a}, 1} \times 10^{7} \\
\text { calculé }\end{array}$ & $\begin{array}{c}\mathrm{D}_{\mathrm{a}}^{*} \times 10^{7} \\
\text { mesuré }\end{array}$ & $\begin{array}{c}\mathrm{E} \\
\text { efficacité }\end{array}$ & $\begin{array}{c}\mathrm{n}\left(\mathrm{Q}_{\mathrm{A}}\right)^{\mathbf{\theta}_{\mathrm{s}}} \\
\text { calculé }\end{array}$ \\
\hline 1 & 0,94 & 0,3 & 0,30 & 0,27 \\
2 & 0,94 & 0,4 & 0,42 & 0,38 \\
3 & 0,94 & 1,0 & 1,05 & 0,67 \\
4 & 0,94 & 1,6 & 1,68 & 1,44 \\
5 & 0,76 & 1,2 & 1,60 & 1,64 \\
6 & 0,69 & 1,0 & 1,45 & 1,73 \\
7 & 0,83 & 0,3 & 0,36 & 0,48 \\
8 & 0,76 & 0,5 & 0,66 & 0,45 \\
9 & 0,66 & 0,5 & 0,76 & 0,42 \\
10 & 0,64 & 3,5 & 5,47 & 5,34 \\
11 & 0,82 & 3,6 & 4,44 & 5,76 \\
12 & 0,82 & 2,8 & 3,45 & 4,60 \\
\hline \hline
\end{tabular}

On aboutit à la formule suivante :

$$
\mathrm{D}_{\mathrm{a}, 3}=\mathrm{D}_{0, \mathrm{P}} \cdot \frac{\theta}{\tau \mathbf{b}^{\prime}} \cdot \mathrm{E} \text {. }
$$

Les résultats calculés pour les 12 expériences précédentes figurent au tableau 4 ; sur la figure 5 est représentée la relation $D^{*}=f\left(D_{a, 3}\right)$, forcée statistiquement à passer par l'origine.

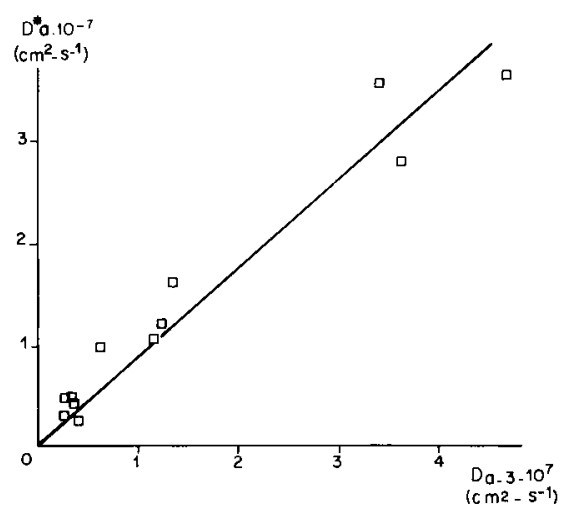

Figure 5

Relation entre les valeurs mesurées du coefficient de diffusion apparent du phosphore et celles prévues par l'équation (21)

Measured values of diffusion coefficient versus data estimated by means of equation (21)

$D_{\mathbf{a}}^{*}=0,87 D_{a, 3} \quad\left(r^{2}=0,95\right)$

\section{DISCUSSION}

Cette étude concernant la diffusivité de l'ion $\mathrm{PO}_{4}^{3-}$ en sols calcaires montre que l'on peut obtenir de deux manières un ordre de grandeur du coefficient de diffusion. Il faut cependant considérer que ces deux méthodes ne sont pas strictement équivalentes.

Une $1^{\text {re }}$ méthode consiste, en utilisant la méthode proposée par HIRA et SING, à déterminer directement un coefficient de diffusion apparent ne découlant que d'une seule expérience dont les paramètres sont fixés par les conditions opératoires ou mesurés a posteriori. Il s'agit bien d'un coefficient apparent, puisque la mesure intègre ces différen- 
tes conditions et que, toutes choses égales d'ailleurs, il suffira d'utiliser ce coefficient dans l'équation :

$$
\frac{\partial \mathrm{C}}{\partial \mathrm{t}}=\mathrm{D}_{\mathrm{a}}^{*} \frac{\partial^{2} \mathrm{C}}{\partial \mathrm{x}^{2}}
$$

pour résoudre le problème du transfert de l'ion $\mathrm{PO}_{4}^{---}$, en admettant toutefois comme négligeable la variation de $D_{a}^{*}$ avec la concentration dans le milieu.

Comme l'ont montré les résultats expérimentaux, ce coefficient $D_{a}^{*}$ dépend, dans une expérience simulant un apport à la surface d'un sol, de la quantité $\mathrm{Q}_{\mathrm{A}}$ apportée. Cette dépendance, incompatible fondamentalement avec la définition d'un coefficient de diffusion (JOST, 1960), s'explique par l'insuffisance du modèle théorique qui ne tient pas compte ni de l'hétérogénéité du plan source, ni du caractère morphologiquement discontinu du contact du sol avec ce plan, ni de l'influence des variations de concentration sur la nature des réactions. Cette hétérogénéité est encore accrue dans les conditions naturelles et rend encore plus aléatoire, l'utilisation de ce coefficient, établi dans les conditions expérimentales proposées par les auteurs.

Une autre méthode consiste à calculer par la formule de NYE (NYE, 1979) un coefficient de diffusion indépendant du niveau de concentration du plan source, à l'aide du coefficient de diffusion dans l'eau en tenant compte de 3 paramètres (les 2 premiers étant phénoménologiques) :

- la tortuosité qui rend compte du trajet physique réel parcouru lors de la diffusion ; on a donné une formule (16) permettant de l'apprécier pour une certaine gamme d'humidité, en connaissant $\rho_{\mathrm{d}}$ et $\rho_{\mathrm{s}}$. Il serait intéressant que des études portant sur un grand nombre de matériaux, dans diverses conditions physiques, permettent d'élargir statistiquement le domaine de validité de cette formule. Par contre, la validité d'un paramètre $\tau$ unique est liée à un domaine de teneurs en eau, variable pour chaque type de sol.

- la relation de partage entre la phase solide et la phase liquide, caractérisée ici par la pente d'un isotherme linéaire d'insolubilisation. L'utilisation du paramètre $\rho_{\mathrm{d}}$ ne dépend que de la forme donnée à cette relation de partage.

- l'humidité volumique qui permet de passer de la concentration dans la solution du sol à la quantité de soluté par unité de volume de sol. On a ainsi :

$$
\mathrm{D}_{\mathrm{a}, 1}=\frac{\theta \cdot \mathrm{D}_{0, \mathrm{P}}}{\tau \mathrm{b} \rho_{\mathrm{d}}} .
$$

L'utilisation du coefficient ainsi obtenu peut alors être envisagé de deux manières:

- en premier lieu, si l'apport est réalisé de façon totalement continu (c'est-à-dire si le sel est dissous dans l'eau d'irrigation), ce coefficient sera utilisé sans modification, dans une équation classique de diffusion mono, -bi- ou tridimensionnelle, si l'on admet que les paramètres $\tau$, b et $\rho_{\mathrm{d}}$ sont constants. Comme $\theta$ dans le cas général est variable, il faudra écrire par exemple :

$$
\frac{\partial \mathrm{C}}{\partial \mathrm{t}}=\frac{\partial}{\partial \mathrm{x}}\left(\mathrm{D}_{\mathrm{a}, 1} \frac{\partial \mathrm{C}}{\partial \mathrm{x}}\right)
$$

en couplant cette équation avec celle qui rend compte des variations de $\theta$ en fonction de $x$. Il faut noter cependant que la quantité de phosphate mise en jeu interviendra, lors des calculs d'intégration numérique, par la condition initiale $\mathrm{C}=\mathrm{C}_{\mathrm{i}}$ (concentration de la solution apportée pour $\mathrm{x}=0$ et $\mathrm{t}=0)$.

- en second lieu, si l'apport consiste en une application au sol sous forme de sels non dissous (et plus particulièrement dans le cas d'un épandage monodimensionnel à sec), on peut également utiliser ce coefficient, pour l'introduire dans l'équation générale de diffusion, mais en tenant compte d'un «facteur de répartition » de l'apport. Pour un sol dont la teneur en eau est inférieure ou égale à la capacité au champ, ce coefficient numérique peut être pris égal à $n Q_{\AA}^{\theta_{s}}$. Pour un sol saturé (ou si l'épandage réalisé est suivi d'un apport d'eau), on pourra admettre que l'on est ramené au cas précédent.

\section{CONCLUSIONS}

On a donc tenté de relier les divers facteurs qui, agissant sur le coefficient de diffusion de l'ion $\mathrm{PO}_{4}^{3-}$, conditionnent sa pénétration par diffusion dans un sol calcaire : linéarité d'un isotherme d'immobilisation, influence de la tortuosité, de la teneur en eau, du mode d'apport ; la formule de NYE permet une discussion du rôle de ces différents paramètres. Les résultats expérimentaux obtenus par la technique de HIRA \& SING confirment la très lente diffusion de l'ion considéré à laquelle pourraient venir s'ajouter ultérieurement des évolutions apatitiques insolubilisant définitivement l'orthophosphate (ARVIEU, 1972). Cette caractéristique liée à la nature calcaire du sol, apparaît en comparant les ordres de grandeur des coefficients $D_{a}^{*}$ (de 0,37 à $3,610^{-7} \mathrm{~cm}^{2} \mathrm{~s}^{-1}$ ) avec ceux mesurés sur un sol argileux non calcaire par HiRA \& SINGH (de 3,3 à $6,910^{-7} \mathrm{~cm}^{2} \mathrm{~s}^{-1}$ pour des humidités volumiques de 0,22 ). Le transfert de $\mathrm{P}$ par diffusion s'accompagne également de la pénétration dans la porosité texturale, phénomène déjà mis en évidence à l'aide d'autoradiographies de ${ }^{32} \mathrm{P}$ par GUNARY (1964): cette pénétration est rendue possible par la lenteur des flux diffusifs.

Corrélativement, la pratique de la ferti-irrigation localisée, remplaçant en partie les flux de diffusion par des flux de transfert convectifs, paraît, en dépit des critiques qui ont été formulées à son égard, plus apte que la fertilisation en plein, par épandage de surface, à assurer une répartition adéquate du phosphore vers les zones d'activité racinaire. Cette répartition doit être réalisée rapidement après l'apport, car l'équilibre que nous avons constaté, pour des temps de contact de l'ordre de $3 \mathrm{j}$, peut évoluer vers une insolubilisation plus poussée. Le cas des sols calcaires peu argileux, où le rôle protecteur de l'argile peut faire défaut (BoUviER, 1974), doit être également considéré. Mais dans ce cas, la conductivité hydraulique plus importante et la tortuosité plus faible peuvent assurer des transferts convectifs plus rapides. 


\section{RÉFÉRENCES BIBLIOGRAPHIQUES}

Arvieu J. C., 1972. Contribution à la connaissance de l'évolution des engrais phosphatés en sol calcaire. Thèse de Doctorat esSciences physiques, Université de Nice, $151 \mathrm{p}$.

Boischot P., Coppenet M., Hebert J., 1950. Fixation de l'acide phosphorique sur le calcaire du sol. Plant Soil, 2, 311-322.

Bouvier O., 1974. Etude expérimentale de laction de la fraction argileuse sur l'évolution du phosphate monocalcique en milieu calcaire. Thèse $3^{\mathrm{c}}$ Cycle, Université de Nice, $109 \mathrm{p}$.

de Camargo O. A., Biggar J. W., Nielsen D. R., 1979. Transport of inorganic phosphorus in an alfisol. Soil Sci. Soc. A.m. J., 43, 884890.

Carslaw H. S., Jaeger J. C., 1947. Conduction of Heat in Solids. Oxford. Clarendon Press. 2nd Edition, $510 \mathrm{p}$.

de Cockborne A. M., 1980. Transfert des nitrates en milieu poreux saturé, en présence de porosité structurale. Thèse Univ., Grenoble, $93 \mathrm{p}$.

Cole C. V., Olsen S. R., Scott C. O., 1953. The nature of phosphate sorption by calcium carbonate. Soil Sci. Soc. Am. Proc., 17, 352-356.

Crank J., 1956. Mathematics of Diffusion. Oxford University Press, Amen House, London E.C. 4, 345 p.

Fathi Amer, Shams M. S., Awad K. M., Khalil M. A., 1980. Immobilization of diammonium phosphate and monocalcium Phosphate in calcareous Soils. Soil Sci. Soc. Am. J., 44, 1174-1178.

Guennelon R., Habib R., de Cockborne A. M., 1979. Aspects particuliers concernant la disponibilité de $\mathrm{N}, \mathrm{P}$ et $\mathrm{K}$ en irrigation localisée fertilisante sur arbres fruitiers. Colloque "Irrigation localisée ». C.E.E., Bologne.

Gunary D., 1964. Phosphate diffusion in intact soil. 8th int. Congress Soil Sci., Bucarest, 573-578.

Hira G. S., Singh N. T., 1977. Observed and predicted rates of phosphorus diffusion in soils of varying bulk density and water content. Soil Sci. Soc. Am. J., 41, 537-540.

Hira G. S., Singh N. T., 1978. Prediction of phosphorus diffusion from fertilizer source. Soil Sci. Soc. Am. J., 42, 561-565.
Jost W., 1960. Diffusion in solids, liquids, gases. Acad. Press Inc., New York 3d Edition, 558 p.

Nakayama F. S., Jackson R. D., 1963. Diffusion of tritiated water (THO) in agar gel and water. J. Phys. Chem., 67, 932.

Nye P. H., 1966. The measurement and mechanism of ion-diffusion in soil. $I$. The relation between self-diffusion and bulk-diffusion. $J$. Soil Sci. 17, (1), 16-23.

Nye P. H., 1968. The use of exchange isotherms to determine diffusion coefficients in soil. 9th int. Congress Soil Sci. Adelaïde, 1, 117-126.

Nye P. H., 1979. Diffusion of ions and uncharged solutes in soils and soil clays. Adv. Agron., 31, 225-272.

O'Neill M. K., Gardner B. R., Roth R. L., 1979. Orthophosphoric acid as a phosphorus fertilizer in trickle irrigation. Soil Sci. Soc. Amer. J., 43, 283-286.

Passioura J. B., 1971. Hydrodynamic dispersion in aggregated media. I. Theory. Soil Sci. Soc. Amer. J., 339-344.

Rauschkolb R. S., Rolston D. E., Miller R. J., Carlton A. B., Burau R. G., 1976. Phosphorus fertilization with drip irrigation. Soil Sci. Soc. Amer. J., 40, 68-72.

Rowell D. L., Martin M. W., Nye P. H., 1967. The measurement and mechanism of ion diffusion in soils. III. The effect of moisture content and soil-solution concentration of self-diffusion of ions in soils. J. Soil Sci., 18 (2), 204-222.

Schofield R. K., Graham-Bryce I. J., 1960. Diffusion in soils. Nature (London), 188, 1048-1049.

Selim H. M., Davidson J. M., Mansell R. S., 1976. Evaluation of a two-site adsorption-desorption model describing solute transport in soil. In Proc. Summer Computer Simulation Conf., Washington, D.C.

Van Genuchten Th., Wierengs P. J., 1976. Mass transfer studies in porous media. I. Analytical solutions. Soil Sci. Soc. Am. J., 40, 473480 . 